\title{
Development of Electrochemical Sensor for Fast Liquor Authentication
}

\author{
Weihong $\mathrm{Wu},{ }^{1 *}$ Mengyao $\mathrm{Wu},{ }^{1}$ Jingtao Zhou, ${ }^{1}$ \\ Yuting $\mathrm{Xu},{ }^{1}$ Zemin $\mathrm{Li},{ }^{1}$ Yuzhe Yao, ${ }^{1}$ and $\mathrm{Li} \mathrm{Fu}^{1,2^{* *}}$ \\ ${ }^{1}$ College of Materials and Environmental Engineering, Hangzhou Dianzi University, Hangzhou 310018, P. R. China \\ ${ }^{2}$ Key Laboratory of Wuliangye-Flavor Liquor Solid-State Fermentation, China National Light Industry, \\ Yibin, Sichuan 644000, China
}

(Received July 1, 2020; accepted July 31, 2020)

Keywords: electrochemical sensor, Chinese liquor, electroactive compounds, food authentication, Wuliangye

In this work, we proposed an electrochemical method for directly recording the profiles of the electroactive compounds in Chinese liquor. We selected samples from 11 brands with four flavor types to investigate the feasibility of the proposed method. Owing to the different raw materials in the fermentation process, different brands of Chinese liquor contain different small molecules that could contribute to differences during an electrochemical scan. Principal component analysis (PCA) and cluster analysis were conducted. The results indicate that the electrochemical profiles of these brands can be distinguished using their voltammetric data. We also selected two distinct peaks corresponding to the electro-oxidation of the electroactive compounds for authentication. The 11 brands were successfully differentiated using a flow chart. In addition, the flow chart has been successfully applied to actual sample analysis.

\section{Introduction}

Chinese liquor has a complex flavor mainly composed of esters. It is made of a starchy material and is prepared by cooking, saccharification, fermentation, distillation, aging, and blending. ${ }^{(1)}$ During its long history of several thousand years, Chinese liquor developed in stages. The first stage was 4000-2000 BC. This stage is known as the enlightenment period of Chinese liquor. Brewing water wine from fermented grains was the main form of brewing at the time. This was the late period of primitive society, when alcohol was regarded as a drink containing great magic. The next stage from $2000 \mathrm{BC}$ to $200 \mathrm{BC}$ was the growth period of Chinese liquor. In this period, with the use of fire and the emergence of grain and livestock, coupled with the invention of yeast, China became the first country in the world to use yeast for wine production. During this period, the winemaking industry developed considerably. The government set up a special institution for making wine, and the wine production was controlled by the government. From $200 \mathrm{BC}$ to $1000 \mathrm{AD}$, the Chinese liquor industry was maturing. During this period, drinking was popular not only among upper classes but also

\footnotetext{
*Corresponding author: e-mail: whwu@hdu.edu.cn

** Corresponding author: e-mail: fuli@hdu.edu.cn

https://doi.org/10.18494/SAM.2020.2972
} 
among common people. During this period, the rise of land trade between China and Europe, other parts of Asia, and Africa resulted in Chinese and western wine cultures permeating each other and laid the foundation for the further development of Chinese liquor.

Owing to differences in geographical and ecological environments, brewing materials, auxiliary materials, types of liquor, and brewing technology, Chinese liquor has different styles and distinct flavors. ${ }^{(2,3)}$ At present, the established flavor types in the liquor industry include nongxiang flavor, jiangxiang flavor, qingxiang flavor, rice flavor, fengxiang flavor, sesame flavor, special flavor, soy flavor, medicinal flavor, and mixed flavor. In this work, we selected the products with nongxiang flavor, jiangxiang flavor, qingxiang flavor, and mixed flavor as examples for study.

Nongxiang flavor liquor is made from sorghum or a variety of grains, and is mixed with high-quality wheat, corn, millet, and rice. Medium- or high-temperature yeast is prepared for solid fermentation in a mud pit for 45-90 days. The aroma of nongxiang flavor liquor is mainly ethyl caproate, supplemented by appropriate amounts of ethyl lactate, ethyl acetate, and ethyl butyrate to form a complex aroma. The grain/yeast ratio of jiangxiang flavor liquor is 1:1 or 1:2. As a saccharifying agent, high-temperature yeast is the precursor of jiangxiang flavoring substances. Jiangxiang flavor liquor has a high acid content and high aldehyde and ketone contents. The contents of n-propanol and heptanol are also high. Qingxiang flavor liquor is characterized by its pure aroma and clean aftertaste. The proportions of ethyl acetate and ethyl lactate in the product are 55 and 45\%, respectively. Mixed flavor contains high heptanoic acid, ethyl heptanoic acid, isoamyl acetate, butyric acid, and isobutyric acid contents.

With the development of science and technology and liquor research in China, researchers have paid increasing attention to the development of liquor testing systems..$^{(4,5)}$ An electronic nose system uses the cross-sensitivity of a sensor array to detect the components of a mixed gas by pattern recognition technology. An electronic tongue detection system is composed of sensor units with high cross-sensitivity to different components in a solution. ${ }^{(6)}$ It is an analytical system that combines appropriate pattern recognition algorithms and multivariate analysis methods to process the array data and obtain qualitative and quantitative information of solution samples. The main components are a sensor array and a signal processing and pattern recognition system. It also includes a potential analysis sensor, a voltammetry analysis sensor, and an optical sensor. Visual array sensor technology has been developed in recent years. It uses a sensor array to detect the sample to be tested and generates a characteristic response signal. Through the signal recognition and processing system, the detection results are displayed in graphs. ${ }^{(7,8)}$

"Fingerprint" technology was first applied to the analysis of traditional Chinese medicine because of the complex mixtures of products in medicines. This technology involves many methods, including thin-layer scanning (TLS), ${ }^{(9)}$ high-performance liquid chromatography (HPLC), ${ }^{(10)}$ gas chromatography (GC) ${ }^{(11)}$ and infrared (IR) spectroscopy. ${ }^{(12)}$ A "fingerprint" refers to a graph that can reflect the individual characteristics of the analysis object stably, accurately, and comprehensively in a fixed analysis method. The application of this method to liquor quality evaluation combined with sensory evaluation will improve the reliability and scientific accuracy of liquor quality evaluation methods, and can also be used to evaluate the 
stability and repeatability of analytical data. An electrochemical recognition system is different from traditional analytical chemistry instruments. Specifically, such a system is concerned not with the concentration of a particular compound but with the overall intensity of the signal generated by the interaction of its different components. The method focuses on detecting the overall feature of differences between samples. Our previous works demonstrated the recording of electrochemical fingerprints of plant samples. ${ }^{(13,14)}$ In this work, we extend the application of electrochemical fingerprints to Chinese liquor authentication.

\section{Materials and Methods}

All liquor samples were purchased from a local supermarket for analysis. Table 1 shows their information and abbreviations. The liquors were used without treatment in the experiments.

All electrochemical determination processes were carried out using a CHI760 electrochemical workstation. A commercial glassy carbon electrode (GCE), a $\mathrm{Ag} / \mathrm{AgCl}$ electrode, and a Pt electrode were used as the working, reference, and counter electrodes, respectively.

A differential pulse voltammetry (DPV) scan was used for electrochemical fingerprint recording. The GCE was polished using alumina slurry after washing in water. Then, the three-electrode system was inserted into a $5 \mathrm{~mL}$ sample. The electrochemical voltammogram recording was conducted at $-0.2-1.2 \mathrm{~V}$ with a pulse amplitude of $50 \mathrm{mV}$, a pulse width of $0.05 \mathrm{~s}$, and a pulse period of $0.5 \mathrm{~s}$.

Fingerprint standardization was carried out to establish quantitative criteria for recognition, where the ratio between the current and the maximum peak current was obtained at different potentials. Principal component analysis (PCA) and cluster analysis were performed on the basis of the recorded electrochemical fingerprint.

Figure 1 shows the schematic process of electrochemical recording fingerprints for 11 brands of Chinese liquors.

\section{Results and Discussion}

Figure 2 shows the electrochemical behavior of the different brands of Chinese liquor. As can be seen from the figure, a very clear electrochemical fingerprint can be obtained by scanning a liquor directly with a GCE. All electrochemical patterns show several distinct

Table 1

Information of all liquor samples.

\begin{tabular}{|c|c|c|c|c|c|}
\hline Name & Abbreviation & Flavor & Name & Abbreviation & Flavor \\
\hline Luzhoulaojiao & $\mathrm{LZ}$ & Nongxiang & Red Star Erguotou & RS & Qingxiang \\
\hline Wuliangye & WL & Nongxiang & Niulanshan & NL & Qingxiang \\
\hline Kouzijiao & $\mathrm{KZ}$ & Mixed & Laymau & LM & Jiangxiang \\
\hline Lang Jiu & LJ & Jiangxiang & Xijiu & $\mathrm{XJ}$ & Jiangxiang \\
\hline Mao-Tai Chiew & MT & Jiangxiang & Baiyunbian & BY & Mixed \\
\hline Tuopai & TP & Nongxiang & & & \\
\hline
\end{tabular}




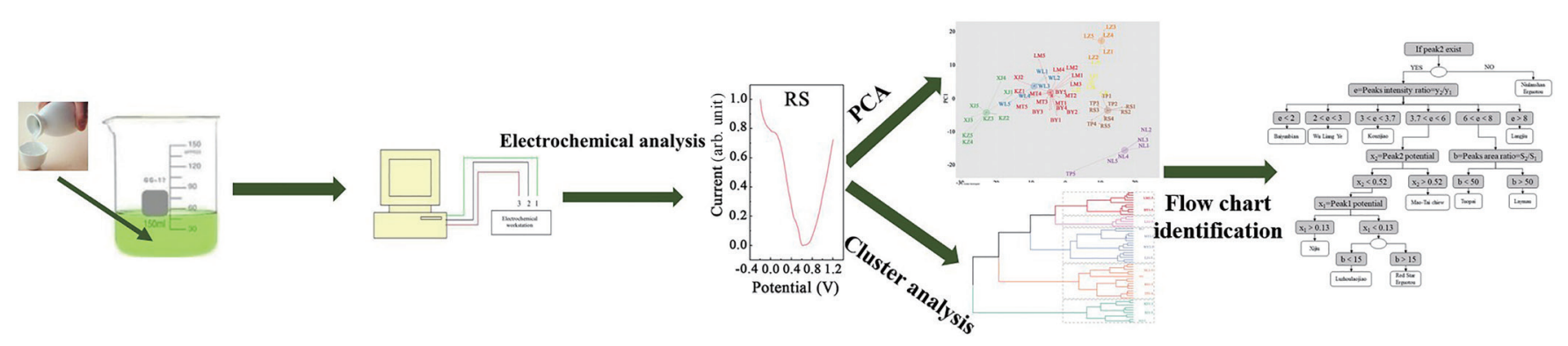

Fig. 1. (Color online) Schematic process of electrochemical recording fingerprints for 11 brands of Chinese liquors.

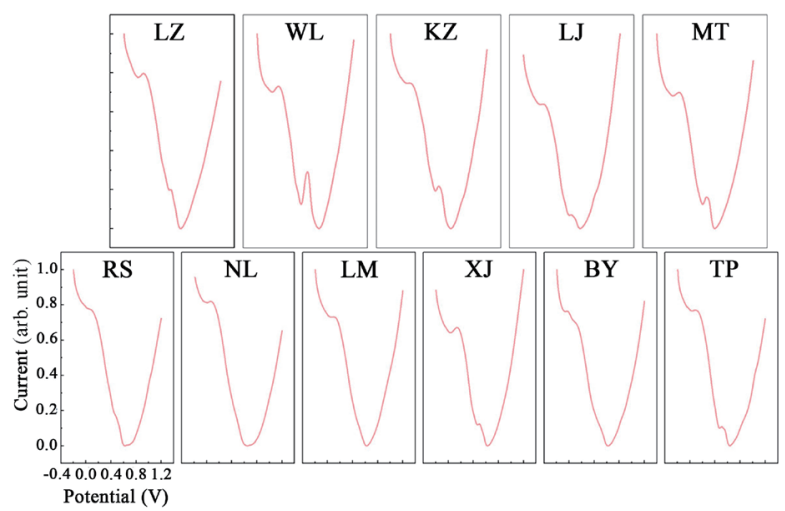

Fig. 2. (Color online) Electrochemical profiles of LZ, WL, KZ, LJ, MT, RS, NL, LM, XJ, BY, and TP.

peaks, representing the oxidation of compounds at these potentials. During the fermentation of liquor, long-chain carbohydrates are broken down to form different small molecules. Some of them have electrochemical activity, so they can be oxidized at lower potentials. Because the raw materials and fermentation processes of different liquors are different, the types and amounts of compounds with electrochemical activity are different in different liquors, so the liquors show different electrochemical behaviors. ${ }^{15,16)}$ Among the samples, except for NL and RS that showed one oxidation peak at $0.15 \mathrm{~V}$, the other samples exhibited two distinct peaks during the scan. However, the potentials of the two peaks differed among the samples. Therefore, the intensity and location of electrochemical oxidation peaks can be used for the identification of Chinese liquor brands.

Before further identification, we first verified the repeatability of the proposed methodology. Figure 3 shows five independent electrochemical fingerprints collected from five bottles for each brand. As can be seen from the figure, our proposed method of directly collecting the electrochemical fingerprint showed good repeatability, and no peak shift was found. Therefore, we believe that this method has practical application.

To observe the difference between the different brands of Chinese liquor statistically, we carried out PCA on the above-mentioned electrochemical fingerprints. As shown in Fig. 4, XJ and $\mathrm{KZ}$ are relatively close, as are LM, WL, BY, and MT. There is also a negligible distance 


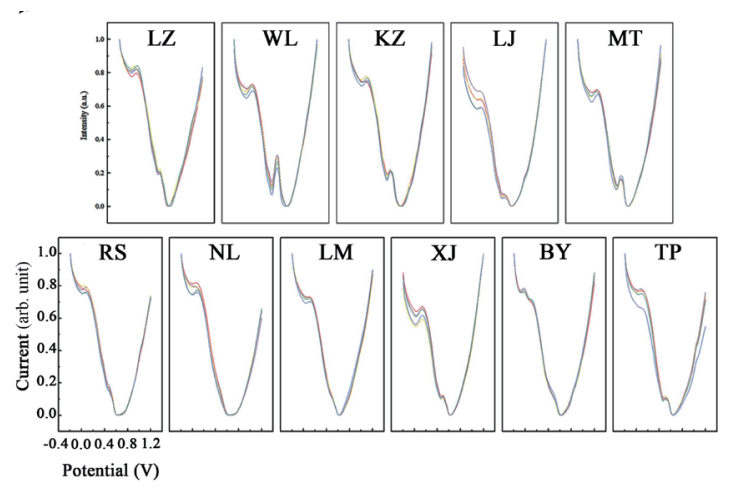

Fig. 3. (Color online) Five independent electrochemical fingerprints collected from five bottles for LZ, WL, KZ, LJ, MT, RS, NL, LM, XJ, $\mathrm{BY}$, and TP.

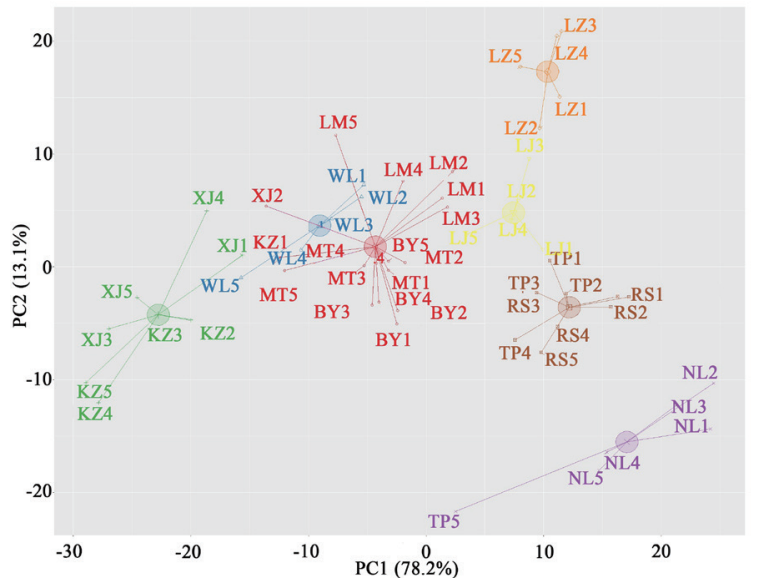

Fig. 4. (Color online) PCA of five electrochemical fingerprints recorded for $\mathrm{LZ}, \mathrm{WL}, \mathrm{KZ}, \mathrm{LJ}, \mathrm{MT}$, RS, NL, LM, XJ, BY, and TP.

between TP and RS. On the other hand, LZ and NL are distant from the other samples. The two factors extracted within the voltammetric data can reach an interpretative capability of more than $90 \%$, suggesting that there were significant differences in electrochemical profiles among the liquors studied in this work.

We also used the cluster analysis method to discriminate between the brands. As shown in Fig. 5, all samples were divided into five clusters. The results were slightly different from those of PCA. Most of the brands were in the bottom cluster. LM and BY were clustered together, in good agreement with the DPV curves recorded in Fig. 1. LZ and NL did not form a cluster with other brands, which is also consistent with Fig. 4. In addition, MT, WL, and LJ were clustered together. These observations indicated that the liquors in one cluster may share similar distributions of electroactive compounds produced during the fermentation. Because the raw materials and fermentation processes of different liquors are different, the types and amounts of compounds with electrochemical activity in different liquors are different and show different electrochemical behaviors. On the basis of the PCA and cluster analysis results, we found that the brands of Chinese liquor can be identified from their DPV profiles.

Figure 6(a) shows the DPV curve of MT. It can be seen that the electrochemical profile of this liquor has two distinct peaks, which are located around 0.1 and $0.5 \mathrm{~V}$. During the liquor fermentation, the long-chain carbohydrates are broken down to form different small molecules. Some of them have electrochemical activity, so they can be oxidized at lower potentials. These oxidation peak potentials vary among the brands. Therefore, they can be used as valuable information for identifying Chinese liquor [Fig. 6(b)]. We also calculated the areas and intensities of these peaks to assist the identification process. Figure 6(c) shows the area ratio between the two peaks for each brand. It can be seen that Laymau had a much higher peak area ratio than the other samples. In contrast, Wuliangye and Baiyunbian had very low peak area ratios. Figure 6(d) shows the peak intensity ratios of all the samples. It can be seen that Laymau, Langjiu, and Tuopai had peak intensity ratios above 6 , while the other samples had values below 6 . 


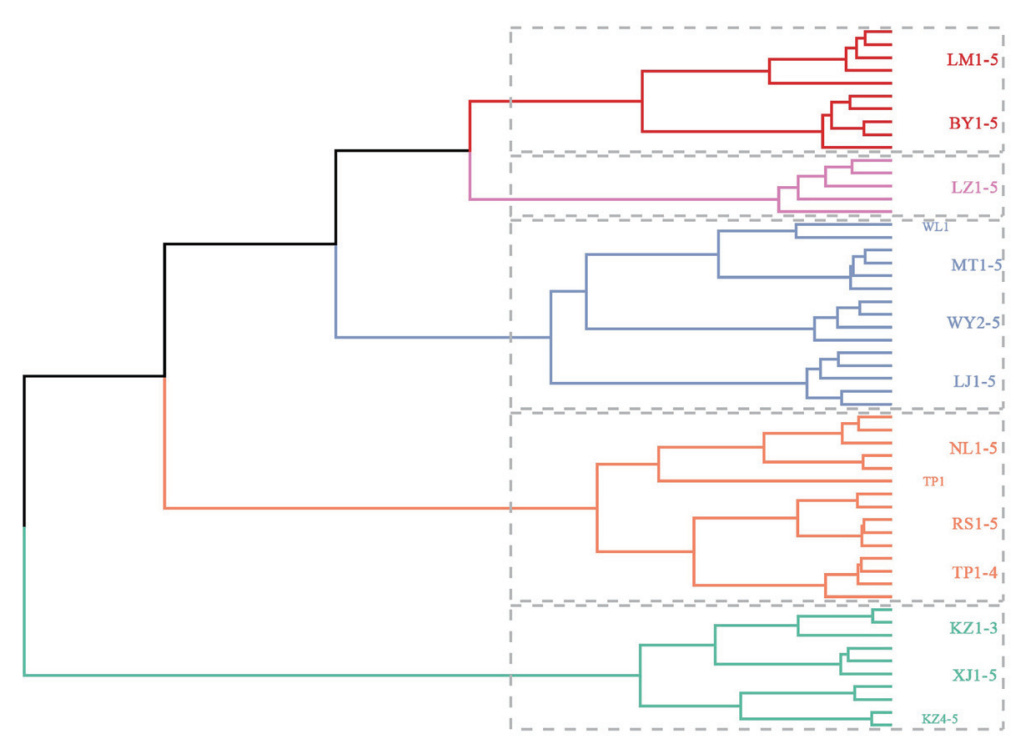

Fig. 5. (Color online) Dendrogram of LZ, WL, KZ, LJ, MT, RS, NL, LM, XJ, BY, and TP based on the electrochemical profiles.

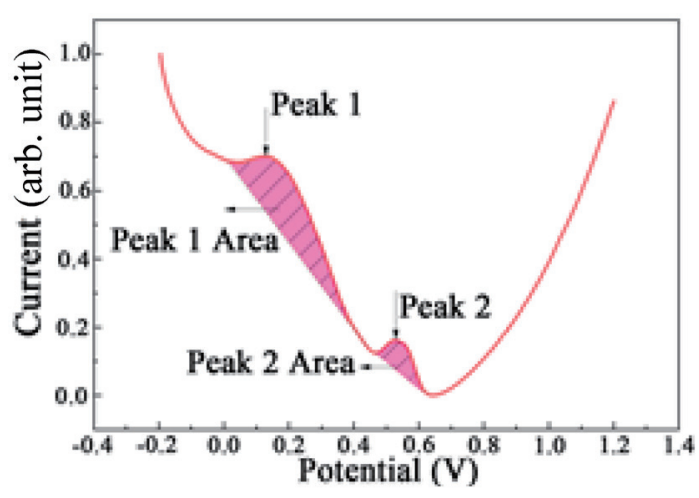

(a)

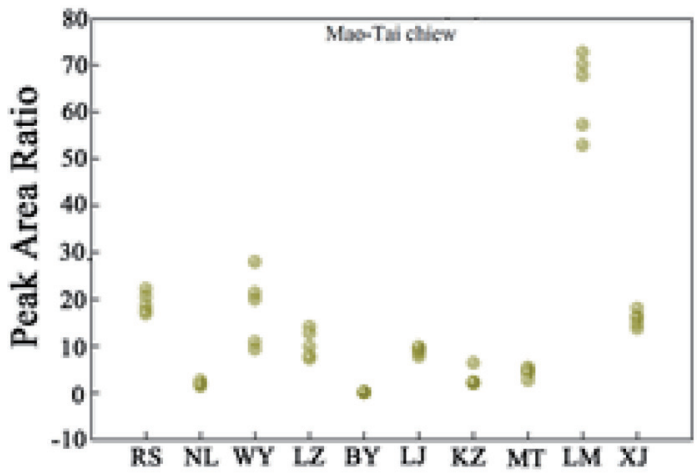

(c)

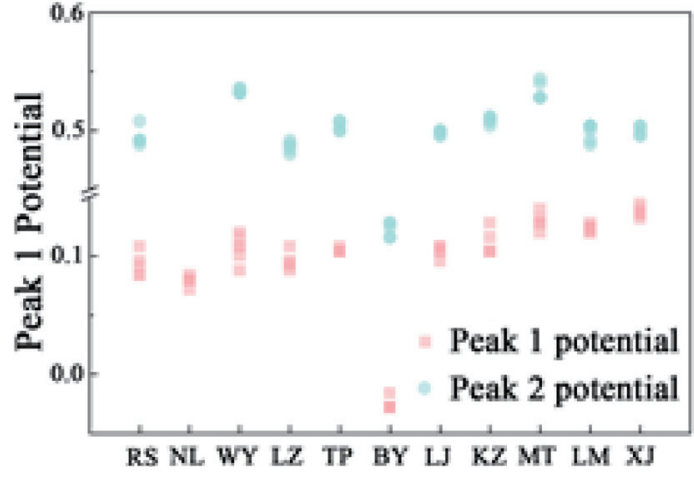

(b)

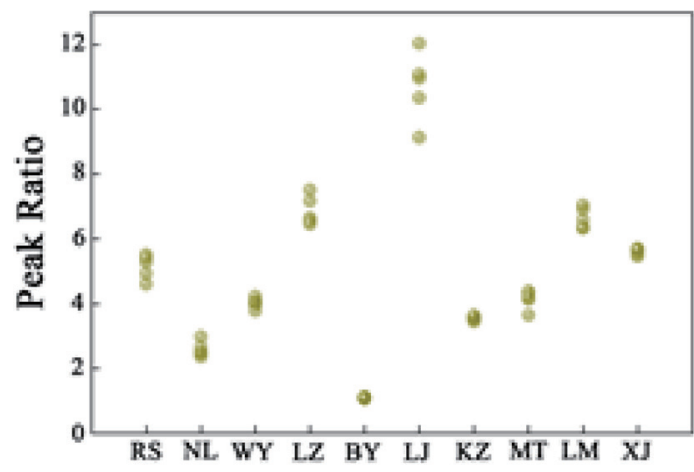

(d)

Fig. 6. (Color online) (a) DPV curve of MT. (b) Peak 1 potential of all Chinese liquor samples. (c) Peak area ratio of all Chinese liquor samples. (d) Peak intensity ratio of all Chinese liquor samples. 


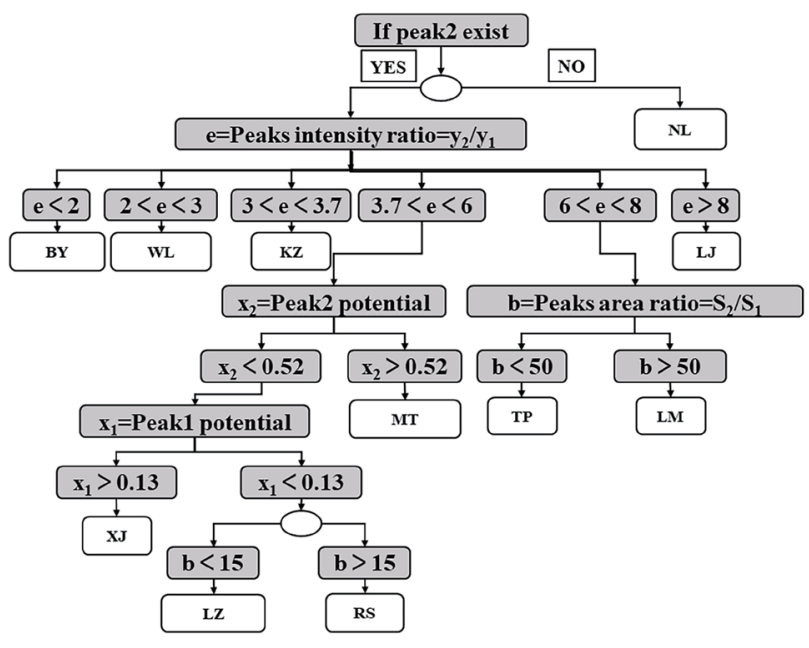

Fig. 7. Authentication flow chart of all Chinese liquor samples.

On the basis of the results deduced from Fig. 6, we proposed a flow chart for brand authentication. As shown in Fig. 7, NL can be identified by the absence of peak 2. Then, the peak intensity ratio is used to identify BY, WL, KZ, and LJ, which have values below 2, between 2 and 3, between 3 and 3.7, and above 8, respectively. If the peak intensity ratio is between 6 and 8, then the peak area ratio is used to identify TP and LM. The peak area ratio of TP is smaller than 50, while that of LM is higher than 50. If the peak intensity ratio is between 3.7 and 6 , the oxidation potential of peak 2 is used to identify MT, whose oxidation potential of peak 2 is higher than $0.52 \mathrm{~V}$. If the oxidation potential of peak 2 is lower than $0.52 \mathrm{~V}$, the oxidation potential of peak 1 is used to identify XJ, whose oxidation potential of peak 1 is higher than $0.13 \mathrm{~V}$. If the oxidation potential of peak 1 is lower than $0.13 \mathrm{~V}$, the peak area ratio is used to identify LZ and RS, which is smaller than 15 for LZ and larger than 15 for RS. We further tested two newly purchased bottles of XJ and LM using the flow chart. The e value of $\mathrm{XJ}$ was 4.42. The $\mathrm{x}_{1}$ of $\mathrm{XJ}$ was 0.50 , whereas that of $\mathrm{LM}$ was 0.21 . The e value of LM was 6.55. The $b$ value was 72 . Both samples can be identified using the flow chart.

\section{Conclusions}

In this work, we proposed an authentication method based on the electrochemical profile of Chinese liquor. A GCE was directly inserted into the liquor sample for electrochemical fingerprint recording. Because of the different raw materials and fermentation processes of different brands of liquor, the types and amounts of compounds and their electrochemical activity are different, resulting in different electrochemical behaviors. PCA and cluster analysis were conducted. The brands of Chinese liquor were successfully identified from their different DPV profiles. 


\section{Acknowledgments}

This project was financially supported by the Key Laboratory of Wuliangye-Flavor Liquor Solid-State Fermentation, China National Light Industry (2018JJ003).

\section{References}

1 P. Wang, Q. Wu, X. Jiang, Z. Wang, J. Tang, and Y. Xu: Int. J. Food Microbiol. 250 (2017) 59. https://doi. org/10.1016/j.ijfoodmicro.2017.03.010

2 Q. Li, Y. Gu, and N. Wang: IEEE Sens. J. 17 (2017) 1788. https://doi.org/10.1109/JSEN.2017.2657653

3 Y. Han, L. Song, S. Liu, N. Zou, Y. Li, Y. Qin, X. Li, and C. Pan: Food Chem. 241 (2018) 258. https://doi. org/10.1016/j.foodchem.2017.08.103

4 L. Chai, P. Xu, W. Qian, X. Zhang, J. Ma, Z. Lu, S. Wang, C. Shen, J. Shi, and Z. Xu: Int. J. Food Microbiol. 297 (2019) 41. https://doi.org/10.1016/j.ijfoodmicro.2019.02.023

5 C. Liu, S. Feng, Q. Wu, H. Huang, Z. Chen, S. Li, and Y. Xu: Front. Microbiol. 10 (2019) 1520. https://doi. org/10.3389/fmicb.2019.01520

6 H. Rottiers, D. A. T. Sosa, L. Van de Vyver, M. Hinneh, H. Everaert, J. De Wever, K. Messens, and K. Dewettinck: Food Anal. Methods 12 (2019) 475. https://doi.org/10.1007/s12161-018-1379-7

7 Z. Shui, J. Li, P. Yang, D. Huo, C. Hou, and C. Shen: Anal. Methods 11 (2019) 5691. https://doi.org/10.1039/ C9AY01791G

8 Y. Huang, P. Cheng, and C. Tan: RSC Adv. 9 (2019) 27583. https://doi.org/10.1039/C9RA05983K

9 L. Peti, P. Augustinus, P. Gadd, and S. Davies: Quat. Int. 514 (2019) 161. https://doi.org/10.1016/ j.quaint.2018.06.039

10 Y. Zhao, X. You, H. Jiang, G. Zou, and B. Wang: J. Chromatogr. B 1104 (2019) 11. https://doi.org/10.1016/ j.jchromb.2018.11.001

11 N. Arroyo-Manzanares, A. Martín-Gómez, N. Jurado-Campos, R. Garrido-Delgado, C. Arce, and L. Arce: Food Chem. 246 (2018) 65. https://doi.org/10.1016/j.foodchem.2017.11.008

12 S. Eid, M. El-Rahman, M. Elghobashy, and K. Kelani: Anal. Chim. Acta 1005 (2018) 70. https://doi. org/10.1016/j.aca.2017.12.011

13 L. Fu, Y. Zheng, P. Zhang, H. Zhang, M. Wu, H. Zhang, A. Wang, W. Su, F. Chen, J. Yu, W. Cai, and C.-T. Lin: Bioelectrochemistry 129 (2019) 199. https://doi.org/10.1016/j.bioelechem.2019.06.001

14 L. Fu, M. Wu, Y. Zheng, P. Zhang, C. Ye, H. Zhang, K. Wang, W. Su, F. Chen, J. Yu, A. Yu, W. Cai, and C.-T. Lin: Sens. Actuators, B 298 (2019) 126836. https://doi.org/10.1016/j.snb.2019.126836

15 L. Norocel and G. Gutt: Aust. J. Grape Wine Res. 25 (2019) 161. https://doi.org/10.1111/ajgw.12375

16 M. Villalonga, B. Borisova, C. Arenas, A. Villalonga, M. Arévalo-Villena, A. Sanchez, J. Pingarrón, A. Briones-Perez, and R. Villalonga: Sens. Actuators B 279 (2019) 15. https://doi.org/10.1016/j.snb.2018.09.092 